\title{
ON THE NUMERICAL RADIUS OF AN ELEMENT OF A NORMED ALGEBRA
}

\author{
by GH. MOCANU
}

(Received 2 April, 1973)

Let $A$ be a unital normed algebra over the complex field $\mathbb{C}, A^{\prime}$ the dual space of $A$, i.e., the Banach space of all continuous linear functionals on $A$, and let $S$ be the set of all states on $A$, i.e.,

$$
S=\left\{s \in A^{\prime} \mid s(1)=1=\|s\|\right\} .
$$

Recall that, for an element $x \in A$, the set

$$
V(x)=\{s(x) \mid s \in S\}
$$

is called the numerical range of $x$, and

$$
v(x)=\sup _{s \in S}|s(x)|
$$

is called the numerical radius of $x$.

We denote by $G(A)$ the set of invertible elements of $A$, by $U_{r}(0)$ the set $\{\lambda \in \mathbb{C}|| \lambda \mid<r\}$, by $\partial U_{r}(0)$ the set $\{\lambda \in \mathbb{C} \| \lambda \mid=r\}$.

We recall also the following assertions.

Lemma. Let $x \in A$. If $v(x)<1$, then

$$
\begin{aligned}
& (1-\lambda x) \in G(A), \\
& \left\|(1-\lambda x)^{-1}\right\| \leqq(1-v(x))^{-1},
\end{aligned}
$$

for all $\lambda \in U_{1}(0)$.

For the proof of this lemma, see [2] or [3].

The aim of this note is to prove the following theorem and to observe that some results concerning the numerical radius are contained in it.

THEOREM. If $x \in A$ and if, for all $\lambda \in \overline{U_{r}(0)}, 1-\lambda x \in G(A)$, then

$$
\left\|x^{n}\right\| \leqq \frac{n !}{r^{n} m(m+1) \ldots(m+n-1)}\left(\sup _{\lambda \in \partial v_{r}(0)}\left\|(1-\lambda x)^{-1}\right\|\right)^{m} \quad(n=1,2, \ldots ; m=1,2, \ldots) .
$$

Proof. We may suppose that $A$ is complete.

From the fact that $1-\lambda x \in G(A)$ for all $\lambda \in \overline{U_{r}(0)}$, it follows that $\rho(r x)<1$, where $\rho$ denotes the spectral radius. Hence the series

$$
1+m \lambda x+\frac{m(m+1)}{2} \lambda^{2} x^{2}+\ldots+\frac{m(m+1) \ldots(m+n-r)}{n !} \lambda^{n} x^{n}+\ldots
$$


converges uniformly on $\overline{U_{r}(0)}$ and

$$
(1-\lambda x)^{-m}=1+m \lambda x+\frac{m(m+1)}{2} \lambda^{2} x^{2}+\ldots+\frac{m(m+1) \ldots(m+n-1)}{n !} \lambda^{n} x^{n}+\ldots
$$

Using the preceding considerations, we deduce that

and therefore

$$
\frac{1}{2 \pi i} \int_{\partial v_{r}(0)} \frac{(1-\lambda x)^{-m}}{\lambda^{n+1}} d \lambda=\frac{m(m+1) \ldots(m+n-1)}{n !} x^{n}
$$

$$
\frac{m(m+1) \ldots(m+n-1)}{n !}\left\|x^{n}\right\| \leqq \frac{1}{r^{n}}\left(\sup _{\lambda \in \partial U_{r}(0)}\left\|(1-\lambda x)^{-1}\right\|\right)^{m} .
$$

This completes the proof.

Corollary 1. (Crabb [4]) For all $x \in A$,

$$
\left\|x^{n}\right\| \leqq n !(e / n)^{n} v(x)^{n} \quad(n=1,2, \ldots) .
$$

Proof. For $m>n$ and $y=n /(m v(x)) x, v(y)=n / m<1$ and from the lemma we deduce that $1-\lambda y \in G(A)$ and $\left\|(1-\lambda y)^{-1}\right\| \leqq(1-n / m)^{-1}$ for all $\lambda \in \overline{U_{1}(0)}$. Hence, using the preceding theorem, we have

$$
\left\|x^{n}\right\| \leqq \frac{m^{n} v(x)^{n}}{n^{n}} \frac{n !}{m(m+1) \ldots(m+n-1)}\left(1-\frac{n}{m}\right)^{-m} .
$$

The assertion follows from this relation on letting $m \rightarrow \infty$.

Corollary 2. (Bohnenblust and Karlin [1]) For all $x \in A$,

$$
e^{-1}\|x\| \leqq v(x) \leqq\|x\| .
$$

COROLlaRy 3. (Stampfli [7]) If $x \in A$ is such that $\rho(x)<2,(1+\lambda x) \in G(A)$ and

for all $\lambda \in \partial U_{1}(0)$, then $x=0$.

$$
\left\|(1+\lambda x)^{-1}\right\| \leqq 1
$$

Proof. If $|\lambda| \leqq \frac{1}{2}$, then $\rho(\lambda x)<1$ and so $1-\lambda x \in G(A)$. If $\frac{1}{2}<|\lambda| \leqq 1$, then we have $\lambda=t \mu$ with $\frac{1}{2}<t \leqq 1,|\mu|=1$ and

$$
1-\lambda x=1-t \mu x=t(1-\mu x)+1-t=t(1-\mu x)\left(1+\{(1-t) / t\}(1-\mu x)^{-1}\right) .
$$

Then $(1-t) / t<1$, and so $\left\|\{(1-t) / t\}(1-\mu x)^{-1}\right\|<1$, which implies that $1-\lambda x$ is invertible.

It follows from the theorem that $x=0$.

Corollary 4. If $x \in A$ and if, for all $\lambda \in U_{1}(0), 1-\lambda x \in G(A)$ and $\left\|(1-\lambda x)^{-1}\right\| \leqq 1$, then $x=0$.

Corollary 5. (See also [6].) Let $A$ be a complex Banach algebra with identity. $A$ is commutative if and only if there exists $K \geqq 1$ such that

$$
x, y \in A \Rightarrow v(x y) \leqq K v(y x) .
$$


From Corollary 2 it follows that $x \rightarrow v(x)$ is a linear norm on $A$ such that

$$
x, y \in A \Rightarrow v(x y) \leqq K\|y x\| \text {. }
$$

From [5, Theorem] it follows that $A$ is commutative.

Corollary 6. (Bonsall and Duncan [3]) Let A be a complex Banach algebra with identity. If there exists $K \geqq 1$ such that

then $A$ is commutative.

$$
x \in A \Rightarrow v(x) \leqq K \rho(x)
$$

This assertion follows from the fact that

$$
x, y \in A \Rightarrow v(x y) \leqq K \rho(x y)=K \rho(y x) \leqq K v(y x) .
$$

COROLLARY 7. Let $A$ be a complex Banach algebra with identity. If

$$
x \in A, s \in \operatorname{Ex} S \Rightarrow s\left(x^{2}\right)=s(x)^{2},
$$

then $A$ is commutative. Here $\operatorname{Ex} S$ denotes the set of all extreme states.

From the fact that

$$
x \in A \Rightarrow|s(x)| \leqq \rho(x),
$$

and the fact that $S=\overline{\operatorname{co}} \operatorname{Ex} S$, it follows that $v(x)=\rho(x)$.

COROLlary 8. Let $A$ be a $C^{*}$-algebra with identity. Then $A$ is commutative if and only if, for any pure state $p$, we have

$$
x \in A \Rightarrow p\left(x^{2}\right)=p(x)^{2} .
$$

\section{REFERENCES}

1. H. F. Bohnenblust and S. Karlin, Geometrical properties of the unit sphere of Banach algebras, Ann. of Math. 62 (1955), 217-229.

2. F. F. Bonsall, The numerical range of an element of a normed algebra, Glasgow Math. J. 10 (1969), 68-72.

3. F. F. Bonsall and J. Duncan, Numerical ranges of operators on normed spaces and of elements of normed algebras, London Math. Soc. Lecture Note Series 2 (Cambridge, 1971).

4. M. J. Crabb, Numerical range estimates for the norms of iterated operators, Glasgow Math. J. 11 (1970), 85-87.

5. Gh. Mocanu, Sur quelques critères de commutativité pour une algèbre de Banach, Analele Univ. Bucuresti Matematică-Mecanică 2 (1971), 127-129.

6. C. Le Page, Sur quelques conditions entrainant la commutativité dans les algèbres de Banach, C.R. Acad. Sci. Paris 265 (1967), 235-237.

7. J. G. Stampfli, An extreme point theorem for inverses in a Banach algebra with identity, Proc. Cambridge Philos. Soc. 63 (1967), 993-994.

\section{FACULTY OF MATHEMATICS}

UNIVERSITY OF BUCHAREST 